\title{
Deterministic Equivalent for the SINR of Regularized Zero-forcing Precoding in Correlated MISO Broadcast Channels with Imperfect CSIT
}

\author{
Sebastian Wagner* ${ }^{* \dagger}$, Romain Couillet ${ }^{\ddagger}$, Mérouane Debbah $^{\ddagger}$, and Dirk T. M. Slock ${ }^{\dagger}$ \\ *ST-ERICSSON, 06560 Sophia-Antipolis, France, Email: \{sebastian.wagner\} @ stericsson.com \\ ${ }^{\dagger}$ EURECOM, 06560 Sophia-Antipolis, France, Email: \{sebastian.wagner, dirk.slock\}@eurecom.fr \\ ${ }^{\ddagger}$ SUPÉLEC, 91192 Gif sur Yvette, France, Email: \{romain.couillet, merouane.debbah\}@ supelec.fr
}

\begin{abstract}
This paper considers the MISO broadcast channel with different spatial correlations of the user vector channels. The base station implements regularized zero-forcing (RZF) precoding based on an imperfect channel estimation. We derive a deterministic equivalent of the signal-to-interference plus noise ratio (SINR) by applying novel results from the field of large dimensional random matrices. Based on this deterministic equivalent, we compute the sum rate maximizing $R Z F$ precoder which is given in closed form for independent and identically distributed channels. Simulations show that the accuracy of the approximated SINR extends well into finite dimensions.
\end{abstract}

\section{INTRODUCTION}

We consider a multiple-input single-output (MISO) broadcast channel where an $M$-antenna base station transmits to $K$ single-antenna users. To mitigate inter-user interference, the data are linearly precoded, based on the available channel state information at the transmitter (CSIT). In this contribution we study regularized zero-forcing (RZF) precoding since it features a good performance-complexity trade-off [1].

This work extends the results in [1]-[3] by applying novel results from the field of large dimensional random matrices. In particular we consider a general channel with per-user correlation and imperfect CSIT. We derive a deterministic equivalent of the signal-to-interference plus noise ratio (SINR) per user. That is, a close approximation independent of the channel realization for every $M$ which is (almost surely) exact as $M \rightarrow \infty$ with finite $M / K$. This approximation can be applied to a wide range of problems [4], [5], among which the present contribution addresses solely the problem of deriving the sum rate maximizing regularization term.

Notation: In the following, boldface lower-case and uppercase characters denote vectors and matrices, respectively. The operators $(\cdot)^{\mathrm{H}}, \operatorname{tr}(\cdot)$ and $E[\cdot]$ denote conjugate transpose, trace and expectation, respectively. The $N \times N$ identity matrix is denoted $\mathbf{I}_{N}, \log (\cdot)$ is the natural $\operatorname{logarithm}$ and $\Im(z)$ is the imaginary part of $z \in \mathbb{C}$.

\section{System Model}

Consider a MISO broadcast channel where a base station (BS) equipped with $M$ antennas transmits to $K$ single-antenna users. The narrow-band signal $y_{k}$ received by user $k$ is

$$
y_{k}=\mathbf{h}_{k}^{\mathrm{H}} \mathbf{x}+n_{k}, \quad k=1,2, \ldots, K
$$

where $\mathbf{h}_{k}^{\mathrm{H}} \in \mathbb{C}^{1 \times M}$ is the channel of user $k, \mathbf{x} \in \mathbb{C}^{M}$ is the transmit vector and the $n_{k}$ are independent complex Gaussian noise terms with zero mean and variance $\sigma^{2}$.

Each user channel $\mathbf{h}_{k}$ is correlated, i.e., $E\left[\mathbf{h}_{k} \mathbf{h}_{k}^{\mathrm{H}}\right]=\boldsymbol{\Theta}_{k}$ and can be written as

$$
\mathbf{h}_{k}=\sqrt{M} \Theta_{k}^{1 / 2} \mathbf{z}_{k}
$$

where $\boldsymbol{\Theta}_{k}$ is the correlation matrix of user $k$ and $\mathbf{z}_{k}$ has i.i.d. complex entries of zero mean and variance $1 / M$. Moreover, only an imperfect estimate $\hat{\mathbf{h}}_{k}$ of the true channel $\mathbf{h}_{k}$ is available at the BS. Following [6], [7], we model $\hat{\mathbf{h}}_{k}$ as

$$
\hat{\mathbf{h}}_{k}=\sqrt{M} \Theta_{k}^{1 / 2}\left(\sqrt{1-\tau_{k}^{2}} \mathbf{z}_{k}+\tau_{k} \mathbf{q}_{k}\right) \triangleq \sqrt{M} \Theta_{k}^{1 / 2} \hat{\mathbf{z}}_{k},
$$

where $\mathbf{q}_{k}$ has i.i.d. entries of zero mean and variance $1 / M$ independent from $\mathbf{z}_{k}$ and $n_{k}$. The parameter $\tau_{k} \in[0,1]$ reflects the amount of uncertainty in the channel estimate $\hat{\mathbf{h}}_{k}$.

The transmit vector $\mathbf{x}$ is a linear combination of the independent user symbols $s_{k} \sim \mathcal{C N}(0,1)$ and can be written as

$$
\mathbf{x}=\sum_{k=1}^{K} \sqrt{p_{k}} \mathbf{g}_{k} s_{k}
$$

where $\mathbf{g}_{k} \in \mathbb{C}^{M}$ and $p_{k} \geq 0$ are the beamforming (BF) vector and the signal power of user $k$, respectively. The BF vectors are normalized to satisfy the average power constraint

$$
E\left[\|\mathbf{x}\|^{2}\right]=\operatorname{tr}\left(\mathbf{P G}^{\mathrm{H}} \mathbf{G}\right) \leq P
$$

where $\mathbf{G} \triangleq\left[\mathbf{g}_{1}, \mathbf{g}_{2}, \ldots, \mathbf{g}_{K}\right] \in \mathbb{C}^{M \times K}, \mathbf{P}=\operatorname{diag}\left(p_{1}, \ldots, p_{K}\right)$ and $P$ is the total available transmit power.

The RZF precoder $\mathbf{G}$ is given by

$$
\mathbf{G}=\xi\left(\hat{\mathbf{H}}^{\mathrm{H}} \hat{\mathbf{H}}+M \alpha \mathbf{I}_{M}\right)^{-1} \hat{\mathbf{H}}^{\mathrm{H}},
$$

where $\hat{\mathbf{H}}^{\mathrm{H}} \triangleq\left[\hat{\mathbf{h}}_{1}, \hat{\mathbf{h}}_{2}, \ldots, \hat{\mathbf{h}}_{K}\right] \in \mathbb{C}^{M \times K}$ is the channel estimate available at the BS, $\xi$ is a normalization scalar to fulfill the power constraint (5) and $\alpha>0$ is the regularization term. Here, $\alpha$ is scaled by $M$ to ensure that, as $M$ grows large, both $\operatorname{tr} \hat{\mathbf{H}}^{\mathrm{H}} \hat{\mathbf{H}}$ and $\operatorname{tr} M \alpha \mathbf{I}_{M}$ grow with the same order of magnitude. 
From the total power constraint (5), we obtain $\xi^{2}$ as

$$
\xi^{2}=\frac{P}{\operatorname{tr} \mathbf{P} \hat{\mathbf{H}}\left(\hat{\mathbf{H}}^{\mathrm{H}} \hat{\mathbf{H}}+M \alpha \mathbf{I}_{M}\right)^{-1} \hat{\mathbf{H}}^{\mathrm{H}}} \triangleq \frac{P}{\Psi} .
$$

Denoting $\rho \triangleq P / \sigma^{2}$ the signal-to-noise ratio (SNR) and $\hat{\mathbf{W}} \triangleq\left(\hat{\mathbf{H}}^{\mathrm{H}} \hat{\mathbf{H}}+M \alpha \mathbf{I}_{M}\right)^{-1}$, the SINR $\gamma_{k}$ of user $k$ under RZF $\mathrm{BF}$ and single-user decoding takes the form

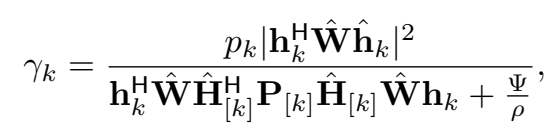

where $\hat{\mathbf{H}}_{[k]}^{\mathrm{H}} \triangleq\left[\hat{\mathbf{h}}_{1}, \ldots, \hat{\mathbf{h}}_{k-1}, \hat{\mathbf{h}}_{k+1}, \ldots, \hat{\mathbf{h}}_{K}\right] \in \mathbb{C}^{M \times K-1}$ and $\mathbf{P}_{[k]} \triangleq \operatorname{diag}\left(p_{1}, \ldots, p_{k-1}, p_{k+1}, \ldots, p_{K}\right)$. The ergodic sum rate is defined as

$$
R_{\text {sum }}=\sum_{k=1}^{K} E_{\hat{\mathbf{H}}}\left[\log \left(1+\gamma_{k}\right)\right] .
$$

\section{DETERMINISTIC EQUIVALENT OF THE SINR}

In this section we derive a deterministic equivalent $\gamma_{k}^{\circ}=$ $\gamma_{k}^{\circ}(M)$ for the SINR $\gamma_{k}$ in (8) such that

$$
\gamma_{k}-\gamma_{k}^{\circ} \stackrel{M \rightarrow \infty}{\longrightarrow} 0
$$

almost surely. That is, $\gamma_{k}^{\circ}$ is an approximation of $\gamma_{k}$ which becomes more accurate as $M$ increases.

In the course of the derivation of $\gamma_{k}^{\circ}$ we require the following theorem.

Theorem 1: Let $\mathbf{B}_{N}=\mathbf{X}_{N}^{\mathrm{H}} \mathbf{X}_{N}+\mathbf{S}_{N}$ with $\mathbf{S}_{N} \in \mathbb{C}^{N \times N}$ Hermitian nonnegative definite and $\mathbf{X}_{N} \in \mathbb{C}^{n \times N}$ random. The $i$ th column $\mathbf{x}_{i}$ of $\mathbf{X}^{\mathrm{H}}$ is $\mathbf{x}_{i}=\mathbf{\Psi}_{i} \mathbf{y}_{i}$, where the entries of $\mathbf{y}_{i} \in \mathbb{C}^{r_{i}}$ are i.i.d. of zero mean, variance $1 / N$ and have finite moment of order $4+\epsilon$, for some $\epsilon>0$. The matrices $\boldsymbol{\Psi}_{i} \in \mathbb{C}^{N \times r_{i}}$ are deterministic. Furthermore, let $\boldsymbol{\Theta}_{i}=\boldsymbol{\Psi}_{i} \boldsymbol{\Psi}_{i}^{\mathrm{H}} \in \mathbb{C}^{N \times N}$ and define $\mathbf{Q}_{N} \in \mathbb{C}^{N \times N}$ deterministic. Both $\boldsymbol{\Theta}_{i}$ and $\mathbf{Q}_{N}$ are assumed to have uniformly bounded spectral norm (with respect to $N$ ). Define

$$
m_{\mathbf{B}_{N}, \mathbf{Q}_{N}}(z) \triangleq \frac{1}{N} \operatorname{tr} \mathbf{Q}_{N}\left(\mathbf{B}_{N}-z \mathbf{I}_{N}\right)^{-1} .
$$

Then, for $z \in \mathbb{C} \backslash \mathbb{R}^{+}$, as $n, N$ grow large with ratios $\beta_{i} \triangleq N / r_{i}$ and $\beta \triangleq N / n$ such that $0<\liminf _{N} \beta(N) \leq \lim \sup _{N} \beta(N)<$ $\infty$, we have that

$$
m_{\mathbf{B}_{N}, \mathbf{Q}_{N}}(z)-m_{\mathbf{B}_{N}, \mathbf{Q}_{N}}^{\circ}(z) \stackrel{N \rightarrow \infty}{\longrightarrow} 0,
$$

almost surely, with $m_{\mathbf{B}_{N}, \mathbf{Q}_{N}}^{\circ}(z)$ given by

$m_{\mathbf{B}_{N}, \mathbf{Q}_{N}}^{\circ}(z)=\frac{1}{N} \operatorname{tr} \mathbf{Q}_{N}\left(\frac{1}{N} \sum_{j=1}^{n} \frac{\boldsymbol{\Theta}_{j}}{1+e_{j}(z)}+\mathbf{S}_{N}-z \mathbf{I}_{N}\right)^{-1}$

where $e_{1}(z), \ldots, e_{n}(z)$ form the unique solution of

$$
e_{i}(z)=\frac{1}{N} \operatorname{tr} \boldsymbol{\Theta}_{i}\left(\frac{1}{N} \sum_{j=1}^{n} \frac{\Theta_{j}}{1+e_{j}(z)}+\mathbf{S}_{N}-z \mathbf{I}_{N}\right)^{-1}
$$

which is the Stieltjes transform of a nonnegative finite measure on $\mathbb{R}^{+}$. Moreover, for $z<0$, the $e_{1}(z), \ldots, e_{n}(z)$ are the unique nonnegative solutions to (14).

Note that (14) forms a system of $n$ coupled equations, from which (13) is given explicitly.

Proof: The proof of Theorem 1 is available in [8].

Based on Theorem 1, the approximated SINR $\gamma_{k}^{\circ}$ is provided in the following theorem.

Theorem 2 (Deterministic equivalent for the SINR): Let $\gamma_{k}$ be the SINR of user $k$ defined in (8). Then

$$
\gamma_{k}-\gamma_{k}^{\circ} \stackrel{M \rightarrow \infty}{\longrightarrow} 0
$$

almost surely, where $\gamma_{k}^{\circ}$ is given by

$$
\gamma_{k}^{\circ}=\frac{p_{k}\left(1-\tau_{k}^{2}\right)\left(m_{k}^{\circ}\right)^{2}}{\Upsilon_{k}^{\circ}\left(1-\tau_{k}^{2}\left[1-\left(1+m_{k}^{\circ}\right)^{2}\right]\right)+\frac{\Psi^{\circ}}{\rho}\left(1+m_{k}^{\circ}\right)^{2}},
$$

where

$$
\begin{aligned}
m_{k}^{\circ} & =\frac{1}{M} \operatorname{tr} \Theta_{k} \mathbf{V}, \\
\Psi^{\circ} & =\frac{1}{M} \sum_{j=1}^{K} \frac{p_{j} e_{j}^{\prime}}{\left(1+e_{j}\right)^{2}}, \\
\Upsilon_{k}^{\circ} & =\frac{1}{M} \sum_{j=1}^{K} \frac{p_{j} e_{j, k}^{\prime}}{\left(1+e_{j}\right)^{2}} .
\end{aligned}
$$

Denoting $\mathbf{V} \triangleq\left(\mathbf{F}+\alpha \mathbf{I}_{M}\right)^{-1}$, three systems of $K$ coupled equations have to be solved. First, the $e_{1}, \ldots, e_{K}$ form the unique positive solutions of

$$
\begin{aligned}
e_{i} & =\frac{1}{M} \operatorname{tr} \boldsymbol{\Theta}_{i} \mathbf{V}, \\
\mathbf{F} & =\frac{1}{M} \sum_{j=1}^{K} \frac{\boldsymbol{\Theta}_{j}}{1+e_{j}} .
\end{aligned}
$$

Secondly, the $e_{1}^{\prime}, \ldots, e_{K}^{\prime}$ form the unique positive solutions of

$$
\begin{aligned}
e_{i}^{\prime} & =\frac{1}{M} \operatorname{tr} \boldsymbol{\Theta}_{i} \mathbf{V}^{2}\left(\mathbf{F}^{\prime}+\mathbf{I}_{M}\right), \\
\mathbf{F}^{\prime} & =\frac{1}{M} \sum_{j=1}^{K} \frac{\boldsymbol{\Theta}_{j} e_{j}^{\prime}}{\left(1+e_{j}\right)^{2}} .
\end{aligned}
$$

Finally, the $e_{1, k}^{\prime}, \ldots, e_{K, k}^{\prime}$ are the unique positive solutions of

$$
\begin{aligned}
e_{i, k}^{\prime} & =\frac{1}{M} \operatorname{tr} \boldsymbol{\Theta}_{i} \mathbf{V}^{2}\left(\mathbf{F}_{k}^{\prime}+\boldsymbol{\Theta}_{k}\right), \\
\mathbf{F}_{k}^{\prime} & =\frac{1}{M} \sum_{j=1}^{K} \frac{\boldsymbol{\Theta}_{j} e_{j, k}^{\prime}}{\left(1+e_{j}\right)^{2}} .
\end{aligned}
$$

Sketch of Proof: Due to space limitations, we can only give a brief sketch of the proof of Theorem 2. For more details refer to a similar proof in [8].

The strategy is as follows: The SINR $\gamma_{k}$ in (8) consists of three terms, (i) the signal power $\left|\mathbf{h}_{k}^{\mathrm{H}} \hat{\mathbf{W}} \hat{\mathbf{h}}_{k}\right|^{2}$, (ii) the interference power $\mathbf{h}_{k}^{\mathrm{H}} \hat{\mathbf{W}} \hat{\mathbf{H}}_{[k]}^{\mathrm{H}} \mathbf{P}_{[k]} \hat{\mathbf{H}}_{[k]} \hat{\mathbf{W}} \mathbf{h}_{k}$ and (iii) the term $\Psi$ of the power normalization. For each of these three terms we will subsequently derive a deterministic equivalent which together constitute the final expression for $\gamma_{k}^{\circ}$. 
a) Deterministic equivalent for $\Psi$ : The term $\Psi=$ $\operatorname{tr} \mathbf{P} \hat{\mathbf{H}}\left(\hat{\mathbf{H}}^{\mathrm{H}} \hat{\mathbf{H}}+M \alpha \mathbf{I}_{M}\right)^{-1} \hat{\mathbf{H}}^{\mathrm{H}}$ can be written as

$$
\begin{aligned}
\Psi & =\sum_{k=1}^{K} p_{k} \hat{\mathbf{h}}_{k}^{\mathrm{H}}\left(\hat{\mathbf{H}}^{\mathrm{H}} \hat{\mathbf{H}}+M \alpha \mathbf{I}_{M}\right)^{-2} \hat{\mathbf{h}}_{k} \\
& \stackrel{(a)}{=} \frac{1}{M} \sum_{k=1}^{K} p_{k} \frac{\hat{\mathbf{z}}_{k}^{\mathrm{H}} \boldsymbol{\Theta}_{k}^{1 / 2} \mathbf{C}_{[k]}^{-2} \boldsymbol{\Theta}_{k}^{1 / 2} \hat{\mathbf{z}}_{k}}{\left(1+\hat{\mathbf{z}}_{k}^{\mathrm{H}} \boldsymbol{\Theta}_{k}^{1 / 2} \mathbf{C}_{[k]}^{-1} \boldsymbol{\Theta}_{k}^{1 / 2} \hat{\mathbf{z}}_{k}\right)^{2}}
\end{aligned}
$$

where $\mathbf{C}_{[k]} \triangleq \boldsymbol{\Gamma}_{[k]}+\alpha \mathbf{I}_{M}$ with $\boldsymbol{\Gamma}_{[k]} \triangleq \frac{1}{M} \hat{\mathbf{H}}_{[k]}^{\mathrm{H}} \hat{\mathbf{H}}_{[k]}$ and in $(a)$ we applied the matrix inversion lemma [9, Lemma 2.2] twice together with (3). For $M$ large, we apply [10, Lemma 2.7] and obtain

$$
\begin{gathered}
\Psi-\frac{1}{M} \sum_{k=1}^{K} p_{k} \frac{\frac{1}{M} \operatorname{tr} \boldsymbol{\Theta}_{k} \mathbf{C}_{[k]}^{-2}}{\left(1+\frac{1}{M} \operatorname{tr} \boldsymbol{\Theta}_{k} \mathbf{C}_{[k]}^{-1}\right)^{2}} \stackrel{M \rightarrow \infty}{\longrightarrow} 0 \\
\stackrel{(b)}{\Leftrightarrow} \Psi-\frac{1}{M} \sum_{k=1}^{K} p_{k} \frac{m_{\Gamma, \Theta_{k}}^{\prime}(-\alpha)}{\left(1+m_{\boldsymbol{\Gamma}, \boldsymbol{\Theta}_{k}}(-\alpha)\right)^{2}} \stackrel{M \rightarrow \infty}{\longrightarrow} 0,
\end{gathered}
$$

almost surely, where in $(b)$ we applied the rank-1 perturbation lemma [11, Lemma 2.1], the definition (11) and denoted $m_{\boldsymbol{\Gamma}, \boldsymbol{\Theta}_{k}}^{\prime}(-\alpha)$ the derivative of $m_{\boldsymbol{\Gamma}, \boldsymbol{\Theta}_{k}}(z)$ along $z$ at $z=$ $-\alpha$. To obtain a deterministic equivalent $m_{\boldsymbol{\Gamma}, \Theta_{k}}^{\circ}(-\alpha)$ and $m_{\boldsymbol{\Gamma}, \boldsymbol{\Theta}_{k}}^{\prime \circ}(-\alpha)$ of $m_{\boldsymbol{\Gamma}, \boldsymbol{\Theta}_{k}}(-\alpha)$ and $m_{\boldsymbol{\Gamma}, \boldsymbol{\Theta}_{k}}^{\prime}(-\alpha)$, respectively, we apply Theorem 1. With the notations in Theorem 2 we have $m_{k}^{\circ} \triangleq m_{\boldsymbol{\Gamma}, \boldsymbol{\Theta}_{k}}^{\circ}(-\alpha)=\frac{1}{M} \operatorname{tr} \boldsymbol{\Theta}_{k} \mathbf{V}$ and $e_{k}^{\prime} \triangleq m_{\boldsymbol{\Gamma}, \boldsymbol{\Theta}_{k}}^{\circ}(-\alpha)=$ $\frac{1}{M} \operatorname{tr} \Theta_{k} \mathbf{V}^{2}\left(\mathbf{F}^{\prime}+\mathbf{I}_{M}\right)$ and therefore

$$
\Psi^{\circ} \triangleq \frac{1}{M} \sum_{k=1}^{K} p_{k} \frac{m_{\Gamma, \Theta_{k}}^{\circ}(-\alpha)}{\left(1+m_{\boldsymbol{\Gamma}, \Theta_{k}}^{\circ}(-\alpha)\right)^{2}}=\frac{1}{M} \sum_{k=1}^{K} \frac{p_{k} e_{k}^{\prime}}{\left(1+e_{k}\right)^{2}},
$$

satisfies $\Psi-\Psi^{\circ} \stackrel{M \rightarrow \infty}{\longrightarrow} 0$, almost surely.

b) Deterministic equivalent for $\mathbf{h}_{k}^{\mathrm{H}} \hat{\mathbf{W}} \hat{\mathbf{h}}_{k}$ : Similar to the derivations in (26) and (27), we have

$$
\begin{aligned}
& \mathbf{h}_{k}^{\mathrm{H}} \hat{\mathbf{W}} \hat{\mathbf{h}}_{k}=\frac{\mathbf{z}_{k}^{\mathrm{H}} \boldsymbol{\Theta}_{k}^{1 / 2} \mathbf{C}_{[k]}^{-1} \boldsymbol{\Theta}_{k}^{1 / 2} \hat{\mathbf{z}}_{k}}{1+\mathbf{z}_{k}^{\mathrm{H}} \boldsymbol{\Theta}_{k}^{1 / 2} \mathbf{C}_{[k]}^{-1} \boldsymbol{\Theta}_{k}^{1 / 2} \hat{\mathbf{z}}_{k}} \\
& =\frac{\sqrt{1-\tau_{k}^{2}} \mathbf{z}_{k}^{\mathrm{H}} \boldsymbol{\Theta}_{k}^{1 / 2} \mathbf{C}_{[k]}^{-1} \boldsymbol{\Theta}_{k}^{1 / 2} \mathbf{z}_{k}}{1+\mathbf{z}_{k}^{\mathrm{H}} \boldsymbol{\Theta}_{k}^{1 / 2} \mathbf{C}_{[k]}^{-1} \boldsymbol{\Theta}_{k}^{1 / 2} \hat{\mathbf{z}}_{k}}+\frac{\tau_{k} \mathbf{z}_{k}^{\mathrm{H}} \boldsymbol{\Theta}_{k}^{1 / 2} \mathbf{C}_{[k]}^{-1} \boldsymbol{\Theta}_{k}^{1 / 2} \mathbf{q}_{k}}{1+\mathbf{z}_{k}^{\mathrm{H}} \boldsymbol{\Theta}_{k}^{1 / 2} \mathbf{C}_{[k]}^{-1} \boldsymbol{\Theta}_{k}^{1 / 2} \hat{\mathbf{z}}_{k}} .
\end{aligned}
$$

Since $\mathbf{q}_{k}$ and $\mathbf{z}_{k}$ are independent, $\mathbf{z}_{k}^{\mathrm{H}} \boldsymbol{\Theta}_{k}^{1 / 2} \mathbf{C}_{[k]}^{-1} \boldsymbol{\Theta}_{k}^{1 / 2} \mathbf{q}_{k} \stackrel{M \rightarrow \infty}{\longrightarrow}$ 0 , almost surely [8] and we obtain

$$
\mathbf{h}_{k}^{\mathrm{H}} \hat{\mathbf{W}} \hat{\mathbf{h}}_{k}-\sqrt{1-\tau_{k}^{2}} \frac{m_{k}^{\circ}}{1+m_{k}^{\circ}} \stackrel{M \rightarrow \infty}{\longrightarrow} 0
$$

almost surely. c) Deterministic equivalent of $\mathbf{h}_{k}^{\mathrm{H}} \hat{\mathbf{W}} \hat{\mathbf{H}}_{[k]}^{\mathrm{H}} \mathbf{P}_{[k]} \hat{\mathbf{H}}_{[k]} \hat{\mathbf{W}} \mathbf{h}_{k}$ : With (2) and $\mathbf{C} \triangleq \Gamma+\alpha \mathbf{I}_{M}, \boldsymbol{\Gamma} \triangleq \frac{1}{M} \hat{\mathbf{H}}^{\mathrm{H}} \hat{\mathbf{H}}$, we have

$$
\begin{aligned}
& \mathbf{h}_{k}^{\mathrm{H}} \hat{\mathbf{W}} \hat{\mathbf{H}}_{[k]}^{\mathrm{H}} \mathbf{P}_{[k]} \hat{\mathbf{H}}_{[k]} \hat{\mathbf{W}}_{k} \\
& =\frac{1}{M} \mathbf{z}_{k}^{\mathrm{H}} \boldsymbol{\Theta}_{k}^{1 / 2} \mathbf{C}^{-1} \hat{\mathbf{H}}_{[k]}^{\mathrm{H}} \mathbf{P}_{[k]} \hat{\mathbf{H}}_{[k]} \mathbf{C}^{-1} \boldsymbol{\Theta}_{k}^{1 / 2} \mathbf{z}_{k} \\
& =\frac{1}{M} \mathbf{z}_{k}^{\mathrm{H}} \boldsymbol{\Theta}_{k}^{1 / 2} \mathbf{C}_{[k]}^{-1} \hat{\mathbf{H}}_{[k]}^{\mathrm{H}} \mathbf{P}_{[k]} \hat{\mathbf{H}}_{[k]} \mathbf{C}^{-1} \boldsymbol{\Theta}_{k}^{1 / 2} \mathbf{z}_{k}+ \\
& \frac{1}{M} \mathbf{z}_{k}^{\mathrm{H}} \mathbf{\Theta}_{k}^{1 / 2}\left[\mathbf{C}^{-1}-\mathbf{C}_{[k]}^{-1}\right] \hat{\mathbf{H}}_{[k]}^{\mathrm{H}} \mathbf{P}_{[k]} \hat{\mathbf{H}}_{[k]} \mathbf{C}^{-1} \boldsymbol{\Theta}_{k}^{1 / 2} \mathbf{z}_{k} .
\end{aligned}
$$

Substituting $\mathbf{C}^{-1}-\mathbf{C}_{[k]}^{-1}=-\mathbf{C}^{-1}\left(\mathbf{C}-\mathbf{C}_{[k]}\right) \mathbf{C}_{[k]}^{-1}$ with $\mathbf{C}-$ $\mathbf{C}_{[k]}=\boldsymbol{\Theta}_{k}^{1 / 2}\left(c_{0} \mathbf{z}_{k} \mathbf{z}_{k}^{\mathrm{H}}+c_{1} \mathbf{q}_{k} \mathbf{q}_{k}^{\mathrm{H}}+c_{2} \mathbf{z}_{k} \mathbf{q}_{k}^{\mathrm{H}}+c_{2} \mathbf{q}_{k} \mathbf{z}_{k}^{\mathrm{H}}\right) \boldsymbol{\Theta}_{k}^{1 / 2}$, where $c_{0} \triangleq\left(1-\tau_{k}^{2}\right), c_{1} \triangleq \tau_{k}^{2}$ and $c_{2} \triangleq \tau_{k} \sqrt{1-\tau_{k}^{2}}$ into (33), we obtain a sum of five terms. Applying [8, Lemma 1] repeatedly to each of these terms yields

$$
\begin{aligned}
& \mathbf{h}_{k}^{\mathrm{H}} \hat{\mathbf{W}} \hat{\mathbf{H}}_{[k]}^{\mathrm{H}} \mathbf{P}_{[k]} \hat{\mathbf{H}}_{[k]} \hat{\mathbf{W}} \mathbf{h}_{k}- \\
& \frac{\Upsilon_{k}\left[1-\tau_{k}^{2}\left(1-\left(1+m_{\boldsymbol{\Gamma}, \boldsymbol{\Theta}_{k}}(-\alpha)\right)^{2}\right)\right]}{\left(1+m_{\boldsymbol{\Gamma}, \boldsymbol{\Theta}_{k}}(-\alpha)\right)^{2}} \stackrel{M \rightarrow \infty}{\longrightarrow} 0,
\end{aligned}
$$

almost surely, where $\Upsilon_{k}=\frac{1}{M^{2}} \operatorname{tr} \mathbf{P} \hat{\mathbf{H}} \mathbf{C}^{-1} \boldsymbol{\Theta}_{k} \mathbf{C}^{-1} \hat{\mathbf{H}}^{\mathrm{H}}$. To find $\Upsilon_{k}^{\circ}$ such that $\Upsilon_{k}-\Upsilon_{k}^{\circ} \stackrel{M \rightarrow \infty}{\longrightarrow} 0$, almost surely, we define $\overline{\mathbf{C}} \triangleq \boldsymbol{\Theta}_{k}^{-1 / 2} \boldsymbol{\Gamma} \boldsymbol{\Theta}_{k}^{-1 / 2}+\alpha \boldsymbol{\Theta}_{k}^{-1}$ and subsequently apply the same techniques as previously. Therefore

$$
\begin{aligned}
\Upsilon_{k}^{\circ} & =\frac{1}{M} \sum_{j=1}^{K} p_{j} \hat{\mathbf{z}}_{j}^{\mathrm{H}} \boldsymbol{\Theta}_{j}^{1 / 2} \boldsymbol{\Theta}_{k}^{-1 / 2} \overline{\mathbf{C}}^{-2} \boldsymbol{\Theta}_{k}^{-1 / 2} \boldsymbol{\Theta}_{j}^{1 / 2} \hat{\mathbf{z}}_{j} \\
& \stackrel{(a)}{=} \frac{1}{M} \sum_{j=1}^{K} p_{j} \frac{m_{\boldsymbol{\Gamma}-z \boldsymbol{\Theta}_{k}, \boldsymbol{\Theta}_{j}}^{\prime \circ}(-\alpha)}{\left(1+m_{\boldsymbol{\Gamma}, \boldsymbol{\Theta}_{j}}^{\circ}(-\alpha)\right)^{2}} \\
& =\frac{1}{M} \sum_{j=1}^{K} \frac{p_{j} e_{j, k}^{\prime}}{\left(1+e_{j}\right)^{2}},
\end{aligned}
$$

where in $(a)$, we applied Theorem 1 to the numerator by setting $\boldsymbol{\Theta}_{j}=\boldsymbol{\Theta}_{k}^{-1 / 2} \boldsymbol{\Theta}_{j} \boldsymbol{\Theta}_{k}^{-1 / 2}$ in the inverse of (13) and (14), which completes the sketch of proof.

In the next section we will discuss one particular application of Theorem 2, namely the sum rate maximizing RZF precoder, for different assumptions on $\boldsymbol{\Theta}_{k}$.

\section{Sum Rate Maximizing Regularization}

The possible applications of Theorem 2 are numerous. One can analyze and optimize e.g. power allocation strategies $\mathbf{P}$, the regularization parameter $\alpha$, the cell loading $M / K$ [4] or CSIT acquisition techniques [5]. In this contribution we focus on the optimization of the regularization parameter $\alpha$.

The objective function is an approximation $R_{\mathrm{sum}}^{\circ}$ of the ergodic sum rate (9), where the instantaneous SINR $\gamma_{k}$ is replaced by its the large system approximation $\gamma_{k}^{\circ}$ in Theorem 2 , i.e.,

$$
R_{\mathrm{sum}}^{\circ}=\sum_{k=1}^{K} \log \left(1+\gamma_{k}^{\circ}\right)
$$

The case $\boldsymbol{\Theta}_{k}=\mathbf{I}_{M}$ is particularly interesting, as it leads to a closed form solution. 
The optimal regularization parameter $\alpha^{\star \circ}$ maximizing (9) is defined as

$$
\alpha^{\star \circ}=\underset{\alpha>0}{\arg \max } \sum_{k=1}^{K} \log \left(1+\gamma_{k}^{\circ}\right) .
$$

The optimization problem (39) is not convex in $\alpha$ and the solution needs to be computed via a one-dimensional line search $^{1}$. Note that for non i.i.d. channels $\left(\boldsymbol{\Theta}_{k} \neq \mathbf{I}_{M}\right)$ the RZF precoder in (6) is not asymptotically optimal anymore.

We refer to the RZF precoder with optimal regularization parameter $\alpha^{\star \circ}$ as ORZF. For homogeneous networks $\left(\boldsymbol{\Theta}_{k}=\mathbf{I}_{M}\right)$ the user channels $\mathbf{h}_{k}$ are statistically equivalent and it is reasonable to assume that the distortions $\tau_{k}^{2}$ of the CSIT $\hat{\mathbf{h}}_{k}$ are identical for all users, i.e., $\tau_{k}^{2}=\tau^{2}$. Under this assumption, it can easily be shown that the power allocation strategy $\mathbf{P}$ maximizing (38) is $\mathbf{P}^{\star \circ}=\frac{P}{K} \mathbf{I}_{K}$, i.e., the total power $P$ is distributed equally among the users. From the above assumptions, the solution to (39) yields a closed form expression for the asymptotically optimal precoder.

Proposition 1: Let $\boldsymbol{\Theta}_{k}=\mathbf{I}_{M}$ and $\tau_{k}=\tau$. The approximated SINR $\gamma_{k}^{\circ}$ of user $k$ under RZF precoding (equivalently, the approximated per-user rate and the sum rate) is maximized for a regularization term $\alpha \triangleq \alpha^{\star \circ}$, given by

$$
\alpha^{\star \circ}=\left(\frac{1+\tau^{2} \rho}{1-\tau^{2}}\right) \frac{1}{\beta \rho} .
$$

Proof: For $\boldsymbol{\Theta}_{k}=\mathbf{I}_{M}, m_{k}^{\circ}=m^{\circ}$ is the Stieltjes transform of the Marčenko-Pastur law and reads [12]

$$
\begin{aligned}
m^{\circ} & =\frac{\beta(1-\alpha)-1+d(\alpha, \beta)}{2 \alpha \beta} \\
\text { with } d(\alpha, \beta) & =\sqrt{\beta^{2} \alpha^{2}+2 \alpha \beta(1+\beta)+(1-\beta)^{2}} .
\end{aligned}
$$

Furthermore, $\Psi^{\circ}\left(m^{\circ}\right)=\Upsilon_{k}^{\circ}=\frac{\frac{P}{K}\left(m^{\circ}\right)^{2}}{\beta\left(1+m^{\circ}\right)^{2}-\left(m^{\circ}\right)^{2}}$. Substituting (41) into (16), differentiating w.r.t. $\alpha$ and equating to zero yields

$$
\left(m^{\circ}\right)^{2}+\frac{1+\frac{1}{\rho}-\beta\left(1-\tau^{2}\right)}{\tau^{2}+\frac{1}{\rho}} m^{\circ}-\frac{\beta\left(1-\tau^{2}\right)}{\tau^{2}+\frac{1}{\rho}}=0 .
$$

As the coefficients of the quadratic polynomial in $m^{\circ}$ are independent of $\alpha$, we solve (42) for $m^{\circ}$ and subsequently find the maximizing $\alpha$. Setting the only positive solution of (42) equal to $m^{\circ}$ in (41) and after some algebraic calculus, we obtain

$$
\frac{\alpha^{2} \beta}{\left(\tau^{2} \rho+1\right)^{2}}\left[\alpha \beta \rho\left(\tau^{2}-1\right)+\tau^{2} \rho+1\right]^{2}=0 .
$$

Since $\alpha>0$, only the term in brackets needs to be considered. The quadratic equation (43) has exactly one distinct real root (40), which completes the proof.

Notice that for perfect CSIT $(\tau=0)$ we have $\alpha^{\star 0}=1 /(\beta \rho)$, which corresponds to the RZF precoder derived in [1], [3]. As mentioned in [1], for large $M$ the RZF precoder is identical to the minimum mean square error (MMSE) precoder in

\footnotetext{
${ }^{1}$ However, in simulations we observe only a single maximum for $\alpha>0$. In this case $\alpha^{\star \circ}$ can be computed very efficiently.
}

[6]. In contrast, for $\tau>0$, the ORZF precoder and the MMSE precoder [6] are not identical anymore, even in the large $M$ limit. Furthermore, for $\tau>0$, at asymptotically high SNR the regularization term $\alpha^{\star 0}$ in (40) converges to $\lim _{\rho \rightarrow \infty} \alpha^{\star \circ}=\frac{\tau^{2}}{1-\tau^{2}} \frac{1}{\beta}$. Thus, for asymptotically high SNR, ORZF is not equivalent to zero-forcing $(\mathrm{ZF})$ precoding, in the sense that $\alpha^{\star \circ}$ does not converge to zero. Similar observations have been made in [6] for the MMSE precoder.

With (40), the approximated SINR $\gamma_{k}^{\circ}$ in (16) takes the following simplified form.

Corollary 1: Let $\boldsymbol{\Theta}_{k}=\mathbf{I}_{M}, \tau_{k}=\tau$ and $\gamma_{k}$ be the SINR of user $k$ under ORZF precoding. Then $\gamma_{k}-\gamma_{k}^{\circ} \stackrel{M \rightarrow \infty}{\longrightarrow} 0$, almost surely, where $\gamma_{k}^{\circ}$ is given by

$$
\gamma_{k}^{\circ} \triangleq \gamma^{\circ}=m^{\circ}\left(-\alpha^{\star \circ}\right)=\frac{\omega}{2} \rho(\beta-1)+\frac{\chi}{2}-\frac{1}{2},
$$

where $\omega \in[0,1]$ and $\chi$ are given by

$$
\begin{aligned}
\omega & =\frac{1-\tau^{2}}{1+\tau^{2} \rho}, \\
\chi & =\sqrt{(\beta-1)^{2} \omega^{2} \rho^{2}+2(1+\beta) \omega \rho+1} .
\end{aligned}
$$

Proof: Replace $\alpha$ in (16) by $\alpha^{\star \circ}$ in (40). After some algebraic manipulations we obtain (44).

Note that for $\tau^{2}=0$ and $\alpha=\alpha^{\star \circ}$, (44) is identical to the asymptotic SINR derived in [3] and for the inter-cell interference-free system in [13]. For $\beta=1$, equation (44) simplifies to $\gamma^{\circ}=-\frac{1}{2}+\sqrt{\omega \rho+\frac{1}{4}}$.

\section{NUMERICAL RESULTS}

In this section we validate our results by comparing them to Monte-Carlo (MC) simulations of i.i.d. Rayleigh blockfading channels for finite system dimensions and equal power allocation $\mathbf{P}=\mathbf{I}_{K}$.

The correlation $\boldsymbol{\Theta}_{k}$ of the $k$ th user channel is modeled as in [14] by assuming a diffuse two-dimensional field of isotropic scatterers around the receivers. The waves impinge the receiver $k$ uniformly at an azimuth angle $\theta$ ranging from $\theta_{k, \min }$ to $\theta_{k, \text { max }}$. Denoting $d_{i j}$ the distance between transmit antenna $i$ and $j$, the correlation is modeled as

$$
\left[\boldsymbol{\Theta}_{k}\right]_{i j}=\frac{1}{\theta_{k, \max }-\theta_{k, \min }} \int_{\theta_{k, \min }}^{\theta_{k, \max }} e^{\mathbf{j} \frac{2 \pi}{\lambda} d_{i j} \cos (\theta)} d \theta
$$

where $\mathbf{j} \triangleq \sqrt{-1}$ and $\lambda$ denotes the signal wavelength. The users are assumed to be distributed uniformly around the BS at an angle $\varphi_{k}=2 \pi k / K$ and as a simple example we choose $\theta_{k, \text { min }}=-\pi$ and $\theta_{k, \max }=\varphi_{k}-\pi$. The $\mathrm{BS}$ is equipped with a uniform linear array (ULA). To assure that $\left\|\Theta_{k}\right\|$ is bounded as $M$ grows large, we assume that the distance between adjacent antennas is independent of $M$, i.e., the length of the ULA increases with $M$.

Figure 1 compares the ergodic sum rate to our deterministic approximation (38). The error bars indicate one standard deviation of the MC results in each direction. The notation $\boldsymbol{\Theta}_{k} \neq \mathbf{I}_{M}$ indicates that $\boldsymbol{\Theta}_{k}$ is modeled according to (47) with $d_{i j} / \lambda=0.5$. It can be observed that the approximation lies 


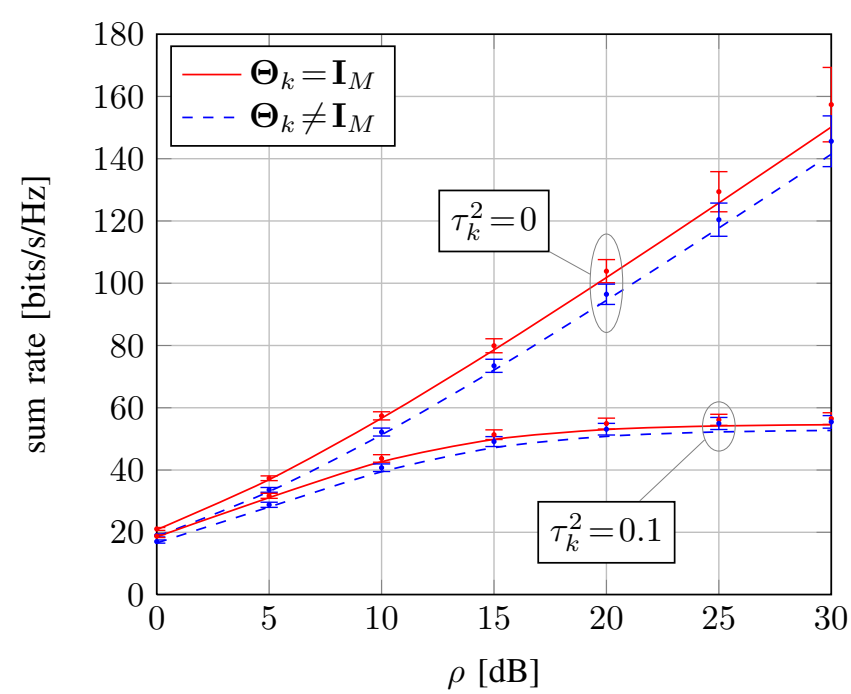

Fig. 1. ORZF, sum rate vs. SNR with $M=K=30$ and $\alpha^{\star 0}$, simulation results are indicated by circle marks with error bars indicating one standard deviation in each direction.

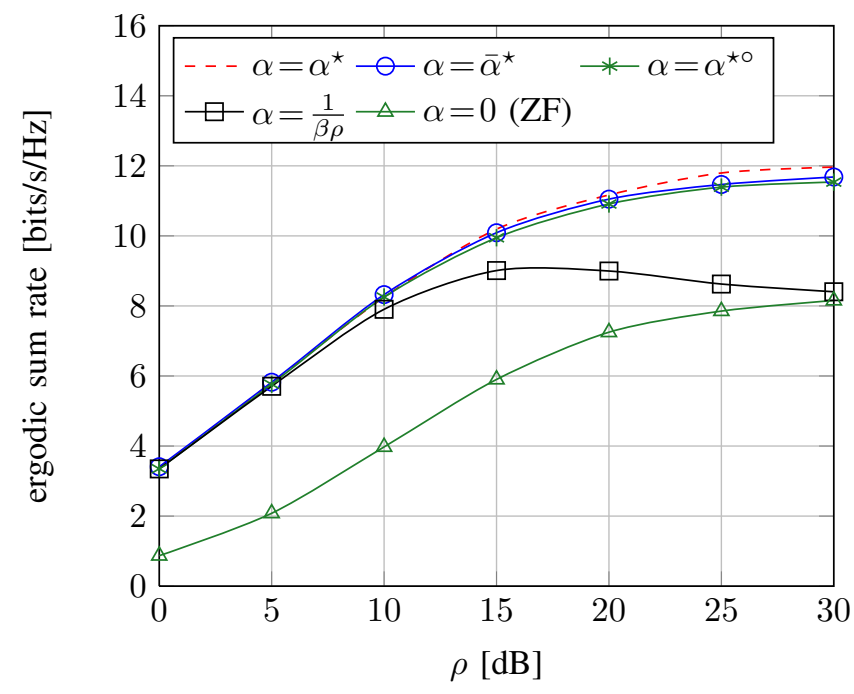

Fig. 2. ORZF, sum rate vs. SNR with $M=K=5, \Theta_{k}=\mathbf{I}_{M}$ and $\tau_{k}^{2}=0.1$.

approximately within the band of two standard deviations of the MC simulations. Therefore, we conclude that the approximation in Theorem 2 is accurate even for finite dimensions and can be applied to various optimization problems.

In Figure 2 we compare the ergodic sum rate performance for different regularization parameters $\alpha$ with CSIT distortion $\tau_{k}^{2}=0.1$. The upper-bound $\alpha=\alpha^{\star}$ is obtained by optimizing $\alpha$ for every channel realization, whereas $\bar{\alpha}^{\star}$ maximizes the ergodic sum rate. It can be observed that both $\bar{\alpha}^{\star}$ and $\alpha^{\star 0}$ perform close to the optimal $\alpha^{\star}$. Furthermore, if the channel uncertainty $\tau_{k}^{2}$ is unknown at the BS (and hence assumed zero), the performance is decreasing as soon as $\tau_{k}^{2}$ dominates the noise power $\sigma^{2}$ and approaches the sum rate of ZF precoding for high SNR. We conclude that (i) adapting the regularization term yields a significant performance increase and (ii) that the proposed ORZF with $\alpha^{\star \circ}$ performs close to optimal even for small system dimensions.

\section{CONCLUSION}

This paper presented a large system approximation of the SINR under RZF precoding for a very general channel model giving rise to numerous possible applications. Among those we discussed the sum rate maximizing RZF precoder and found that an optimization based on the novel deterministic equivalent achieves an almost optimal performance even for small system dimensions.

\section{REFERENCES}

[1] C. B. Peel, B. M. Hochwald, and A. L. Swindlehurst, "A VectorPerturbation Technique for Near-Capacity Multiantenna Multiuser Communication-Part I: Channel Inversion and Regularization," IEEE Trans. Commun., vol. 53, no. 1, pp. 195-202, Jan. 2005.

[2] R. Couillet, S. Wagner, M. Debbah, and A. Silva, "The Space Frontier: Physical Limits of Multiple Antenna Information Transfer," in Proceedings of the 3rd ACM International Conference on Performance Evaluation Methodologies and Tools (VALUETOOLS'08), no. 84, Athens, Greece, Oct. 2008.

[3] V. K. Nguyen and J. S. Evans, "Multiuser Transmit Beamforming via Regularized Channel Inversion: A Large System Analysis," in Proc. IEEE Global Communications Conference (GC'08), New Orleans, LO, Dec. 2008, pp. 1-4.

[4] S. Wagner, R. Couillet, D. T. M. Slock, and M. Debbah, "Large System Analysis of Zero-Forcing Precoding in MISO Broadcast Channels with Limited Feedback," in Proc. IEEE International Workshop on Signal Processing Advances for Wireless Communications (SPAWC'10), Marrakech, Marocco, Jun. 2010.

[5] S. Wagner, R. Couillet, M. Debbah, and D. T. M. Slock, "Optimal Training in Large TDD Multi-user Downlink Systems under Zeroforcing and Regularized Zero-forcing Precoding," in Proc. IEEE Global Communications Conference (GC'10), Miami, USA, Dec. 2010, pp. 1-5.

[6] A. D. Dabbagh and D. J. Love, "Multiple Antenna MMSE Based Downlink Precoding with Quantized Feedback or Channel Mismatch," IEEE Trans. Commun., vol. 56, no. 11, pp. 1859-1868, Nov. 2008.

[7] T. Yoo and A. Goldsmith, "Capacity and Power Allocation for Fading MIMO Channels with Channel Estimation Error," IEEE Trans. Inf. Theory, vol. 52, no. 5, pp. 2203-2214, May 2006.

[8] S. Wagner, R. Couillet, M. Debbah, and D. T. M. Slock, "Large System Analysis of Linear Precoding in MISO Broadcast Channels with Limited Feedback," IEEE Trans. Inf. Theory, submitted for publication. [Online]. Available: http://arxiv.org/abs/0906.3682

[9] J. W. Silverstein and Z. D. Bai, "On the Empirical Distribution of Eigenvalues of a Class of Large Dimensional Random Matrices," Journal of Multivariate Analysis, vol. 54, no. 2, pp. 175-192, Aug. 1995.

[10] Z. D. Bai and J. W. Silverstein, "No Eigenvalues Outside the Support of the Limiting Spectral Distribution of Large Dimensional Sample Covariance Matrices," Annals of Probability, vol. 26, no. 1, pp. 316-345, Jan. 1998

[11] _ "On the Signal-to-Interference Ratio of CDMA Systems in Wireless Communications," Annals of Applied Probability, vol. 17, no. 1, pp. 81-101, Feb. 2007.

[12] A. M. Tulino and S. Verdú, Random Matrix Theory and Wireless Communications. Delft, Netherlands: Now Publishers Inc., 2004.

[13] R. Zakhour and S. V. Hanly, "Base station cooperation on the downlink: Large system analysis," IEEE Trans. Inf. Theory, Jun. 2010. [Online]. Available: http://arxiv.org/abs/1006.3360

[14] W. C. Jakes and D. C. Cox, Microwave Mobile Communications. Hoboken, NJ: Wiley-IEEE Press, 1994. 